\section{Comunidades y sitios de memoria: metodología para la puesta en valor de los sitios de memoria}

\author{
Isabel Donetch Bravo \\ ORCID: https://orcid.org/0000-0002-6679-9148 \\ University College London, Londres, Reino Unido. \\ Correo electrónico: isabel.bravo.19@ucl.ac.uk
}

Resumen

Este artículo busca presentar un nuevo enfoque para la puesta en valor del patrimonio memorial de Chile. El artículo propone que los valores, en cuanto constructos sociales, son subjetivos y cambiantes y que, en el caso de los sitios de memoria, son altamente contestados. A partir de un caso de estudio específico, el Parque por la Paz Villa Grimaldi, se analizó cómo diferentes comunidades valoraban el sitio. Para ello, se emplearon múltiples métodos para la colección de datos: una revisión bibliográfica exhaustiva, el estudio de documentos oficiales del sitio y del Consejo de Monumentos Nacionales, también de reseñas online dejadas por los visitantes. Estas fueron examinadas a través de un análisis temático cualitativo, usando como punto de partida los valores propuestos por Worthing y Bond (2008). A partir de este primer análisis, se definió que los valores son interdependientes unos de otros y que la significancia del sitio opera como sistema complejo y multidimensional. Utilizando un enfoque de sistema dinámico, se mapearon los valores, ilustrando las relaciones entre valores y comunidades. El artículo concluye que, para asegurar la sustentabilidad de los sitios de memoria a futuro, es necesaria la inclusión de las perspectivas de los diferentes actores sociales en la puesta en valor de estos. Asimismo, el artículo ofrece recomendaciones para gestionar potenciales tensiones y desarrollar una estrategia de puesta en valor integrada y holística.

Palabras clave

Comunidades, sitios de memoria, valores, Villa Grimaldi

\section{Communities and Sites of Memory: A methodology for the valuation of Sites of Memory}

\begin{abstract}
This article seeks to introduce a novel approach for the valuation of the memorial heritage of Chile. The paper proposes that values, as social constructs, are subjective and changing and that, in the case of Sites of Memory, they are highly contested. We analyzed how different communities valued the site based on a specific case study, the Parque por la Paz Villa Grimaldi. For this, we used multiple methods for data collection: an exhaustive bibliographic review, the review of official documents of the site and the Council of National Monuments, and online reviews. These were analyzed through qualitative thematic analysis, using as initial themes the values proposed by Worthing and Bond. From this first analysis, we defined that the values are interdependent and that the site's significance operates as a complex and multidimensional system. Values mapped using a dynamic system approach illustrate the relationships between values and communities. The article concludes that to ensure the sustainability of the Memory Sites in the future, it is necessary to include the perspectives of the different social actors in their enhancement. Furthermore, the article offers recommendations for managing potential tensions and developing an integrated and holistic enhancement strategy.
\end{abstract}

\section{Keywords}

Communities, Sites of Memory, values, Villa Grimaldi 


\section{Introducción}

En las últimas décadas, tanto en Chile como en otros países que vivenciaron la violencia y la represión estatal, se ha consolidado un campo de estudio con respecto a los lugares ocupados como centros de detención, tortura y exterminio como parte del patrimonio cultural de una sociedad (Cabeza, 2017; Fernández et al., 2018). Este interés se suma a los cambios en la percepción del concepto mismo de patrimonio cultural, el cual se ha movido desde una visión centrada en lo monumental y excepcional a una basada en los valores que las comunidades les atribuyen a estos bienes (Seguel, 2017). En otras palabras, aquello que legitima el carácter patrimonial de un objeto, tangible o intangible y que, por ende, hace que valga la pena su conservación, son los significados y valores que las diversas comunidades le otorgan a este (Clark, 2010; Díaz-Andreu, 2017).

En este contexto, el reconocimiento de los lugares relacionados con las violaciones a los derechos humanos durante la dictadura cívico-militar en Chile (19731990) como sitios de memoria y su declaratoria como monumentos históricos ha marcado estos espacios como parte del patrimonio nacional y, en consecuencia, se reconoce que poseen cierto valor para la sociedad. Dichos valores son establecidos en los decretos de declaratoria otorgados por el Ministerio de las Culturas, las Artes y el Patrimonio a través del Consejo de Monumentos Nacionales (CMN). Al respecto, a pesar de que diversos autores afirman que los valores de un bien patrimonial varían de comunidad en comunidad y a través del tiempo, llama la atención que estas declaratorias resultan ser muchas veces definitivas, mostrando poca o nula modificación a lo largo de los años. De acuerdo con la nómina de monumentos nacionales desarrollada por el CMN en 2021, de los 1.743 reconocidos solo el $5 \%$ cuenta con modificaciones a sus declaratorias originales, de las cuales solo una corresponde a un sitio de memoria: el Campo de Prisioneros Tres y Cuatro Álamos. Este antiguo centro de detención y tortura fue declarado monumento histórico en el año 2012 y, por solicitud de la comunidad de memoria del sitio, el decreto de declaratoria fue modificado en el año 2017 donde se ampliaron sus límites (en un comienzo la declaratoria incluía solo algunos edificios y ahora comprende el sitio en su totalidad) y se incorporaron nuevos atributos y valores, los cuales son significativos para comprender su relevancia en cuanto sitio de memoria.

Entendiendo entonces que los valores son cambiantes y subjetivos, al estudiar cómo las diferentes comunidades se relacionan con los sitios de memoria surgen múltiples interrogantes, tales como: ¿cómo se valorizan estos sitios?, ¿quiénes valoran estos lugares? Y más importante aún, ¿para quiénes se valorizan y protegen estos sitios? Estudiar cómo se relacionan con sus múltiples comunidades es, por tanto, un aspecto clave en la construcción de memorias en un espectro más amplio de la sociedad, que va más allá de las funciones de reparación simbólica hacia las víctimas que estos sitios cumplen (Fernández et al., 2018). Por lo mismo, este artículo busca estudiar cómo las diferentes comunidades valoran los sitios de memoria, argumentado que una mayor inclusión de actores sociales en la puesta en valor de estos espacios, puede traer beneficios en la construcción de memorias en otras comunidades, promoviendo el interés y participación de los diversos grupos, mejorando la gestión del sitio y asegurando su sustentabilidad a futuro (Avrami et al., 2019).

Para ello, se estudió un caso en particular: el Parque por la Paz Villa Grimaldi, el cual es uno de los sitios de memoria más significativos en Chile y en el cono sur, tanto por ser "uno de los principales lugares de detención y tortura de la DINA" (Lazzara, 2003, p. 128) como por ser el primer espacio de memoria en Latinoamérica recuperado por la comunidad - proceso que no estuvo exento de tensiones (Dalla Porta y Gatica, 2016) -. El caso de Villa Grimaldi es además representativo, porque fue en primera instancia valorado por la comunidad de memoria y los vecinos que lucharon por la recuperación del sitio (Salazar y Artaza, 2013) y, posteriormente, por el Estado en el año 2004. Las diferencias temporales en la puesta en valor por parte de las diferentes comunidades reflejan las tensiones al valorizar el patrimonio, proceso que, según Smith (2021), es influenciado por los discursos con respecto a este y, por lo tanto, es extremadamente politizado, emocional y contextual, entre otras características. Asimismo, la 
importancia de Villa Grimaldi como sitio de memoria y como bien patrimonial se evidencia al ser de uno de los que más visitas recibe anualmente (en el año 2019, por ejemplo, recibió 28.312 visitas, mientras que a Londres 38 fueron 25.504 personas (Corporación Parque por la Paz Villa Grimaldi [CPPVG], 2019; Londres 38, 2019). No obstante, a pesar de su reconocimiento como parte del patrimonio cultural, la puesta en valor del Parque por la Paz Villa Grimaldi no ha estado exenta de tensiones (Dalla Porta y Gatica, 2016). Por lo mismo este artículo busca explorar cómo las diversas comunidades valoran y se relacionan con el sitio.

\section{Marco teórico}

\section{De los lugares a los sitios de memoria}

El término lugar de memoria, acuñado por Pierre Nora, define a estos lugares como espacios físicos o simbólicos, que permiten acceder al pasado (Nora, 1984, citado en Montenegro et al., 2015). Según Nora, los lugares de memoria "son, ante todo, restos, la forma extrema bajo la cual subsiste una conciencia conmemorativa (...). Los lugares de memoria nacen y viven del sentimiento de que no hay memoria espontánea" (Nora, 2009, p. 24). A partir de esta definición, es posible inferir dos cosas: 1) los lugares de memoria hablan de un cierto pasado y 2) como la memoria es un proceso frágil, los lugares de memoria son construidos para conservar una narrativa del pasado para las futuras generaciones. De este modo, estos sitios son espacios que preservan y a la vez gatillan la memoria de una comunidad (Hartley, 2012). En sociedades que han experimentado eventos traumáticos, resulta esencial proteger la memoria de las violaciones a los derechos humanos, que al recordar lo ocurrido y reconocer el sufrimiento de las víctimas se reduce la posibilidad de que actos como estos se repitan en el futuro (Piper et al., 2018; Whigham, 2017).

Tras las horribles consecuencias de los conflictos armados del siglo XX, el concepto de lugar de memoria fue reemplazado por el de sitio de memoria, para definir a aquellos lugares físicos investidos con significación cultural debido a lo ocurrido en ellos en el pasado (International
Coalition of Sites of Conscience [ICSC], 2018). En estos sitios, el valor asociativo es fundamental y, en el caso de Latinoamérica, está vinculado principalmente con las violaciones a los derechos humanos cometidas durante las dictaduras cívico-militares de la segunda mitad del siglo XX. En el contexto latinoamericano, la patrimonialización de los sitios de memoria ha sido parte de las denominadas politicas del pasado y memoria (Seguel, 2018), la cuales son entendidas como las acciones desarrolladas por los Estados

abocadas al reconocimiento estatal de los hechos y de su responsabilidad por las graves violaciones a los derechos humanos ocurridas, la reivindicación y conservación de la memoria histórica y a la promoción de una cultura de los derechos humanos y democracia orientada a la no repetición de los hechos (Comisión Interamericana de Derechos Humanos, 2019, pp. 3-4).

Existe cierto consenso regional por reconocer y proteger sitios de memoria pues estos: 1) aportan información invaluable para recuperar y transmitir la verdad de lo ocurrido en dichos lugares; 2) sirven como evidencia probatoria en los procesos judiciales en curso o que puedan abrirse a futuro; 3) son herramientas para la construcción de memorias vinculadas con crímenes de Estado, brindando espacios para la concientización y educación en torno a los derechos humanos, como parte de las garantías de no repetición y de reparación simbólica (Instituto de Políticas Públicas en Derechos Humanos del Mercosur [IPPDH], 2012).

A nivel nacional, las políticas de memoria son refrendadas por los informes de las Comisiones de Verdad y Reconciliación (Seguel, 2018). Específicamente en el informe de la Comisión Nacional Sobre Prisión Política y Tortura (CNPPT, 2005) se estipula la necesidad de declarar "los principales centros de tortura como monumentos nacionales (...) que puedan servir el propósito de reconocimiento de lo sucedido y compromiso con el respeto de la dignidad de las personas” (p. 528). Sin embargo, a pesar de las medidas de reparación simbólica estipuladas en estos informes, a la fecha de los 1.165 recintos reconocidos en el informe de la CNPPT solo un 
Figura 1

Comunidades de un sitio de memoria

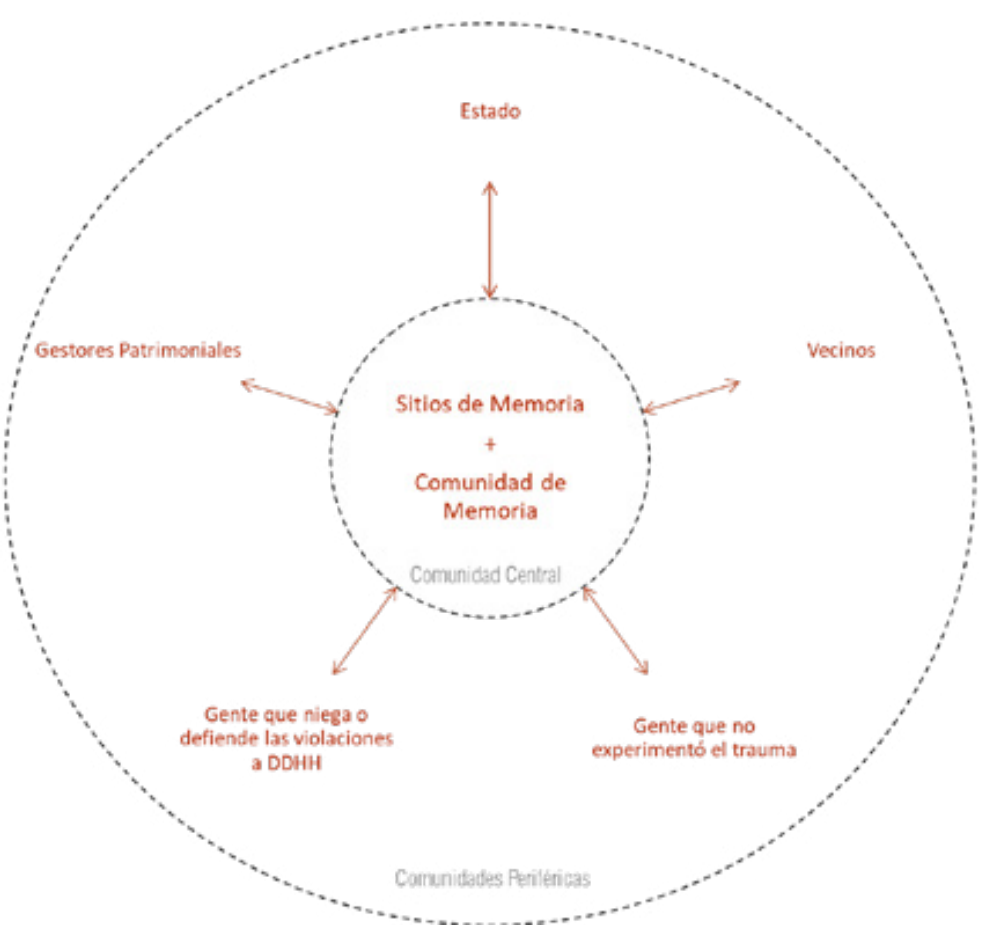

$2,9 \%$ de estos cuenta con protección patrimonial. Esto, según Seguel (2018), está dado por el espíritu de la Ley sobre Monumentos Nacionales, la cual "genera en la práctica una serie de trabas y dificultades, tanto para la recuperación de los sitios, como para la identificación, financiamiento, gestión y puesta en valor de los mismos" (p. 78). Se entiende, por ende, que los sitios de memoria corresponden a un tipo de patrimonio complejo y en tensión, donde diversos actores y discursos se relacionan y disputan.

Asimismo, se propone el entendimiento de los sitios de memoria como espacios sostenidos por tres pilares: una memoria (Bianchini, 2014; Montenegro et al., 2015); una o más comunidades que conmemoran en él (Bustamante, 2016; González, 2018); y los valores que estas le adscriben al lugar (Rosemberg, 2011; Sevcenko, 2010). Bajo esta premisa, un sitio de memoria es el resultado de una acción permanente e intencionada por una o varias comunidades, donde "su valor es contraído según las circunstancias del presente, y sus marcas son significativas para una determinada memoria colectiva" (Fernández et al., 2018, p. 3). Estos significados no se establecen una vez, sino que son dinámicos y cambiantes, los cuales se inscriben en los sitios de memoria, pero también se problematizan, tensionan y cuestionan. Estas discrepancias en la valorización es lo que Tunbridge y Ashworth (1996) llaman la disonancia del patrimonio, la cual, si bien es inherente a todo tipo de bien patrimonial (Kisić, 2016; Smith, 2006), es especialmente notoria en espacios con una alta connotación política y social como son los sitios de memoria.

\section{Sitios de memoria y comunidades}

Con respecto a las comunidades en torno a un sitio de memoria, múltiples autores las han clasificado de diversas maneras, ya sea por rango etario (Sepúlveda et al., 2015) o por cómo se relacionan con el pasado y su memoria (Stern, 2004). Sin embargo, al estudiar los sitios de memoria en cuanto patrimonio cultural, vale la pena el análisis de sus comunidades, como propone Ioannis Poulios (2014), según la relación que tengan con el sitio. Poulios clasifica a las comunidades en centrales y periféricas (Figura 1). Una comunidad central "no solo participa en el proceso patrimonial, sino que se empodera de este; tiene la habilidad de establecer la agenda, tomar decisiones y mantener el poder durante todo el proceso" 
(Poulios, 2014, p. 23). Diversos autores se refieren a la comunidad central de un sitio de memoria como comunidad de memoria (Bianchini, 2015, Del Valle, 2018), la cual usualmente está constituida por los sobrevivientes y los familiares de las víctimas (Bustamante, 2016), que valoran estos lugares y exigen su reconocimiento y protección con el objetivo de transmitir una memoria en particular (Reyes et al., 2016). Son aquellos que fueron directamente afectados por las perpetraciones cometidas en el sitio y actúan tanto como usuarios como custodios del espacio y su memoria.

No obstante, para asegurar la conservación y transmisión del sitio y su memoria a futuro, las comunidades de memoria deben relacionarse con las comunidades periféricas, involucrándolas, fortaleciendo la identidad de la sociedad en su conjunto (Del Valle, 2018). Una de las comunidades con la que se vinculan estrechamente es el Estado. Desde el retorno a la democracia ha sido responsabilidad de este desarrollar políticas de memoria que establezcan la salvaguarda de estos sitios como una iniciativa de reconocimiento de la verdad y reparación simbólica a las víctimas (IPPDH, 2012). Estas políticas, sin bien son consideradas insuficientes por muchos, proveen de cierta protección legal a los sitios de memoria y otorgan, en ciertos casos, aportes financieros para su conservación y gestión. Además, sientan las bases para que los profesionales que trabajan en estos lugares puedan desarrollar programas culturales, educacionales y sociales que promuevan una interpretación más inclusiva, facilitando una identificación empática de parte de los visitantes con el sitio y su memoria, preservando así su valor testimonial.

Por otra parte, al estar emplazado en un territorio geográfico y social específico, cada sitio de memoria se relaciona también con la comunidad local. Es imprescindible comprender este "vínculo inquebrantable" entre los sitios de memoria y su territorio a la hora de desarrollar la puesta en valor, pues este tendrá una incidencia directa en la vida de sus vecinos. Por el contrario, una comunidad en constante tensión con los sitios de memoria la componen aquellas personas que niegan o justifican las atrocidades del pasado. Según
Stern (2004), la memoria colectiva de esta comunidad se basa en el recuerdo del golpe de estado de 1973 como un nuevo comienzo que salvó al país del desastre o de una "memoria de salvación". Esta comunidad difícilmente reconoce valores en los sitios de memoria por el valor asociativo que estos encarnan, argumentando que estos solo muestran "una versión del pasado", la cual ha sido políticamente manipulada.

Por último, otra comunidad relevante a tener en consideración son las personas que no experimentaron el trauma, ya sea porque durante la época de represión no sufrieron directamente la violencia o porque son parte de las nuevas generaciones. Estas últimas son la audiencia principal de los sitios de memoria (Gugglielmucci, 2018), pues al no haber experimentado directamente los horrores del pasado poseen mayor curiosidad sobre este $y$, a su vez, una visión más crítica de sus consecuencias en el presente (Fernández et al., 2018).

\section{Conceptualizando los valores en los sitios de memoria}

El concepto de valor ha sido definido por Avrami y Mason (2019) como "las cualidades, características, significados, percepciones o asociaciones adscritas a los objetos que deseamos conservar" (p. 11). Asimismo, Jameson (2008, citado en McClelland et al., 2013) argumenta que los valores en cuanto atributos otorgados a un bien promueven conexiones intelectuales y emocionales, las cuales justifican la significancia que un bien dado tiene para una comunidad. Sin embargo, los valores no debiesen ser entendidos solo como las cualidades valoradas sino también como las fuerzas o procesos que moldean estas características. Por lo mismo, estos deben comprenderse como procesos dinámicos (Fouseki, \& Sakka, 2013), socialmente construidos (Worthing, \& Bond, 2008) y que, por ende, varían de persona en persona y a través del tiempo (Mason, 2002).

Por esta razón, la valorización de un bien patrimonial puede resultar bastante compleja e incluso un proceso en constante tensión. Por ejemplo, puede ser que un sitio presente múltiples estratos históricos o cualidades 
o, como en el caso de los sitios de memoria, puede ser objeto de disputa entre las diversas comunidades que buscan sanar los traumas del pasado y aquellos grupos que pretenden preservar la memoria hegemónica de quienes están en posiciones de poder (Korostelina, 2019). Por lo mismo, para lidiar con las controversias y disonancias del patrimonio cultural memorial es importante identificar a los diversos actores sociales (o comunidades) y entender cómo son sus relaciones con el sitio, las cuales pueden ser tanto íntimas como conflictivas (Avrami et al., 2000). En el caso de Villa Grimaldi, si bien han existido casos interesantes que intentaron involucrar a las diferentes comunidades en la puesta en valor del sitio y su historia (Rebolledo y Sagredo, 2020; Sagredo, 2019), en los documentos donde se señala su significancia -tales como los planes de gestión- poco se habla de los valores, y cuando ocurre es desde la perspectiva de la comunidad de memoria y de los trabajadores del sitio.

\section{Metodología}

\section{Diseño metodológico}

Este estudio busca reflexionar en torno a cómo se valorizan los sitios de memoria en Chile. Para ello, la investigación, de naturaleza exploratoria, utiliza una metodología cualitativa y un caso de estudio único: el Parque por la Paz Villa Grimaldi, pues resulta ser un caso representativo de la situación de los sitios de memoria en Chile, debido a su importancia tanto histórica como social, ya que fue "uno de los sitios más grandes y comandados por la DINA, de carácter neurálgico en la represión que se llevó a cabo (...) entre los años 1974 y 1978" (López, 2013, p. 53), y el primer sitio de este tipo en ser recuperado por la comunidad en Latinoamérica. En primer lugar, se realizó una revisión exhaustiva de la bibliografía con respecto a puesta en valor, sitios de memoria y sobre el caso de estudio en cuestión. Por otro lado, considerando la actual crisis sanitaria del COVID-19 y, por ende, la imposibilidad de visitar el sitio o de utilizar métodos presenciales para la recolección de datos, se decidió utilizar información de acceso público para llegar a las formas en las que las diferentes comunidades lo evalúan. Los datos analizados son especificados en la Tabla 1. Estas múltiples fuentes fueron usadas para lograr la triangulación de datos y, por tanto, mejorar su validez. Los datos recolectados fueron estudiados mediante un análisis temático cualitativo, un método cualitativo muy utilizado para identificar los temas (es decir, valores) más recurrentes en los datos (Van der Hoeven, 2020).

\section{Procedimiento analítico}

Se realizó un análisis temático, pues este ayuda a organizar y describir datos detalladamente (Braun, \& Clarke, 2006) y presenta el análisis a través de "redes temáticas" (AttrideStirling, 2001, citado en Rishi, \& Gaur, 2012, p. 133). El análisis temático fue desarrollado en cuatro fases. En la primera, se definieron los temas con respecto a la puesta en valor de Villa Grimaldi. Para ello se identificaron valores a partir de la revisión bibliográfica, utilizando finalmente aquellos propuestos por Worthing y Bond (2008), ya que, dentro de las múltiples tipologías de valorización del patrimonio cultural estudiadas, esta reconoce un amplio espectro posibilidades, siendo una de las metodologías más holísticas e inclusivas (Tabla 4). En la fase dos, cada set de datos fue compilado en una base de datos electrónica utilizando el software NVivo 12. Estos fueron codificados temáticamente según los valores especificados, incluyendo además la categoría "sin valor", la cual corresponde a la connotación de que el sitio no posee valores para ser considerado parte del patrimonio cultural de Chile. A partir del análisis de datos se clasificaron los valores en cuatro tipologías según su relevancia para los diferentes actores. Estas categorías se detallan en la Tabla 5. Con base en los resultados de esta fase, una red temática fue desarrollada en la fase tres la cual, a través de un enfoque de sistema dinámico (Newton, \& Fouseki, 2018), busca mapear "las relaciones dinámicas de las variables que configuran un fenómeno, sistema, comportamiento, etc.” (p. 531). De este modo la información codificada en el análisis temático se presenta a través de un diagrama que ilustra las relaciones entre valores y comunidades. Este enfoque permite comprender la significancia de un sitio de memoria como un sistema complejo y multidimensional, el cual 
Tabla 1

Comunidades analizadas y materiales utilizados para su estudio

\begin{tabular}{lll}
\hline Comunidad & Material de investigación & Descripción \\
\hline $\begin{array}{l}\text { Comunidad de memoria. } \\
\text { Gestores del sitio. }\end{array}$ & $\begin{array}{l}\text { Planificación estratégica de Villa Grimaldi 2018- } \\
2028 \text { (CPPVG, 2018). }\end{array}$ & Plan de Gestión de Villa Grimaldi. \\
\hline Estado. & $\begin{array}{l}\text { Declaratoria de Villa Grimaldi otorgada por el } \\
\text { CMN. }\end{array}$ & Decreto N $^{\circ} 264$ (2004). \\
\hline $\begin{array}{l}\text { Gente que no experimentó el trauma. } \\
\text { Gente que justifica lo sucedido en el sitio. }\end{array}$ & $\begin{array}{l}\text { Reseñas online en plataformas como TripAdvisor, } \\
\text { Google Maps y Facebook. }\end{array}$ & $\begin{array}{l}458 \text { reseñas fueron analizadas (ver Tabla 2 y Tabla } \\
3 \text { ). }\end{array}$ \\
\hline
\end{tabular}

Tabla 2

Total de reseñas por plataforma

\begin{tabular}{lll}
\hline Plataforma & $\mathbf{N}^{\circ}$ de reseñas & Puntuación total \\
\hline Facebook & 59 & 4,8 \\
\hline Google Maps & 262 & 4,7 \\
\hline TripAdvisor & 137 & 4,4 \\
\hline Total & 458 & 4,6 \\
\hline
\end{tabular}

Tabla 3

Información demográfica de reseñas

\begin{tabular}{|c|c|c|c|c|c|}
\hline Demografía & Facebook & Google Maps & TripAdvisor & Total & Total relativo \\
\hline \multicolumn{6}{|l|}{ Género } \\
\hline Hombre & 22 & 130 & 47 & 199 & $43,6 \%$ \\
\hline Mujer & 35 & 118 & 62 & 215 & $47,1 \%$ \\
\hline S/especificar & 1 & 14 & 27 & 42 & $9,2 \%$ \\
\hline \multicolumn{6}{|l|}{ Idioma } \\
\hline Español & 58 & 254 & 104 & 416 & $91,2 \%$ \\
\hline Inglés & 1 & 5 & 4 & 10 & $2,2 \%$ \\
\hline Portugués & 0 & 0 & 22 & 22 & $4,8 \%$ \\
\hline Francés & 0 & 0 & 4 & 4 & $0,9 \%$ \\
\hline Italiano & 0 & 3 & 2 & 5 & $1,1 \%$ \\
\hline
\end{tabular}

Tabla 4

Comparación entre tipologías de valorización.

\begin{tabular}{|c|c|c|}
\hline Riegl (1903) & Australia ICOMOS (2013) & Mason (2002) \\
\hline $\begin{array}{ll}\text { - } & \text { Edad } \\
\text { - } & \text { Conmemorativo } \\
\text { - } & \text { Uso } \\
\text { - } & \text { Novedad }\end{array}$ & $\begin{array}{ll}\text { - } & \text { Estético } \\
\text { - } & \text { Histórico } \\
\text { - } & \text { Científico } \\
\text { - } & \text { Social } \\
\text { - } & \text { Espiritual }\end{array}$ & $\begin{array}{ll}\text { - } & \text { Histórico } \\
\dot{*} & \text { Cultural/simbólico } \\
\text { - } & \text { Social } \\
\text { - } & \text { Espiritual } \\
\dot{\text { - }} & \text { Estético } \\
& \text { Económico } \\
\end{array}$ \\
\hline English Heritage (2008) & Worthing y Bond (2008) & \\
\hline $\begin{array}{ll}\text { - } & \text { Testimonial } \\
\text { - } & \text { Histórico } \\
\text { - } & \text { Estético } \\
\text { - } & \text { Comunal/Social }\end{array}$ & $\begin{array}{ll}\text { - } & \text { Estético } \\
\text { - } & \text { Escénico } \\
\text { - } & \text { Arquitectónico } \\
\text { - } & \text { Histórico } \\
\text { - } & \text { Asociativo } \\
\text { - } & \text { Arqueológico } \\
\text { - } & \text { Económico } \\
\text { - } & \text { Educacional } \\
\text { - } & \text { Recreacional }\end{array}$ & $\begin{array}{ll}\text { - } & \text { Artístico } \\
\text { - } & \text { Social } \\
\text { - } & \text { Conmemorativo } \\
\text { - } & \text { Simbólico } \\
\text { - } & \text { Espiritual } \\
\text { - } & \text { Inspiracional } \\
\text { - } & \text { Ecológico } \\
\text { - } & \text { Medioambiental }\end{array}$ \\
\hline
\end{tabular}

Nota: Tabla realizada con base en Worthing y Bond (2008). 
Tabla 5

Categorías de valores identificados en análisis temático

\begin{tabular}{lll}
\hline Clasificación & Definición & Valores \\
\hline Valores centrales & Valores consensuados de Villa Grimaldi. & $\begin{array}{l}\text { Histórico, asociativo, educacional, conmemorativo, } \\
\text { simbólico. }\end{array}$ \\
\hline Valores disonantes & $\begin{array}{l}\text { Valores en tensión entre las diferentes } \\
\text { comunidades. }\end{array}$ & Estético, social, sin valor. \\
\hline Valores secundarios & Valores con un bajo índice de reconocimiento. & $\begin{array}{l}\text { Inspiracional, espiritual, recreacional, } \\
\text { medioambiental, arqueológico, escénico. }\end{array}$ \\
\hline Valores no considerados & Valores no reconocidos por ninguna comunidad. & Económico, ecológico, artístico, arquitectónico. \\
\hline
\end{tabular}

Figura 2

Referencias según valor y actor socia

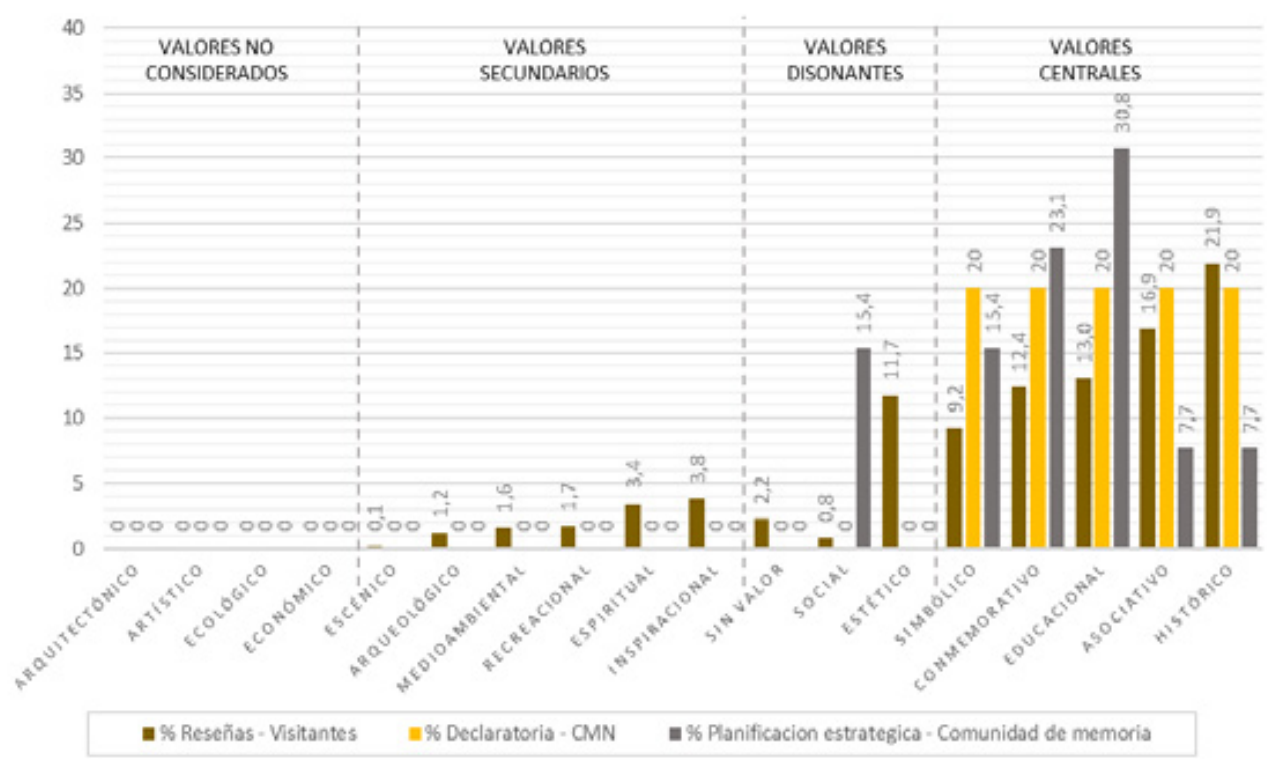

dependerá de todos sus elementos para continuar en el tiempo (Fouseki, \& Nicolau, 2018). Por último, el estudio ofrece recomendaciones para mejorar las metodologías de puesta en valor de sitios de memoria, las cuales deben ser inclusivas e intercomunitarias.

\section{Limitaciones}

El uso de reseñas en línea presenta ciertas limitaciones para este estudio, las cuales deber ser explicitadas. Dado que las reseñas responden a una pregunta específica (¿por qué recomiendas (o no) este lugar?), la información proporcionada y recopilada a partir de estas representa solo una pequeña fracción de la experiencia general de cada visitante. Sin embargo, dado que el reseñar un sitio es opcional, se asume que las respuestas reflejan la percepción de estos y es representativa de su perspectiva. Con respecto al uso de la declaratoria de Monumento Nacional otorgada por el CMN, este documento posee más de 15 años y, por lo mismo, podría no ser representativo de la visión del Estado en la actualidad con respecto a los valores de Villa Grimaldi. Sin embargo, este es el único documento oficial que señala la visión institucional del Estado chileno.

\section{Resultados}

En esta sección se discutirán las diferentes categorías de valores que emergieron del análisis de los valores en Villa Grimaldi. Al estudiar en detalle la forma en que las diversas comunidades valorizaban el sitio es posible ver que la significación de Villa Grimaldi no es uniforme y que más bien varía de comunidad en comunidad (Figura 2). En este sentido, de la amplia gama inicial es posible observar que existen algunos que no son reconocidos por ninguna de las comunidades, los cuales serán relevados más adelante. 
A continuación, se discutirán tres de las clasificaciones de valores identificadas: 1 ) valores centrales: valores consensuados de Villa Grimaldi; 2) valores disonantes: valores en tensión entre las diferentes comunidades; 3) valores secundarios: valores con un bajo índice de reconocimiento.

\section{Valores centrales}

Se habla de valores centrales cuando estos son ampliamente reconocidos por las diversas comunidades. Es decir, donde hay un cierto consenso acerca de los valores que el sitio encarna, siendo evidenciados por todas las comunidades estudiadas. Dentro de los valores centrales de Villa Grimaldi se encuentran el valor histórico, asociativo, educacional, conmemorativo y simbólico (o político). Si bien, se aprecia un aparente consenso, estos no son evaluados de igual manera por las diferentes comunidades. En primer lugar, llama la atención que el valor histórico, definido por la habilidad que tiene el lugar para conectar con personas y eventos del pasado (English Heritage, 2008; Mason, 2002) es ampliamente reconocido por los visitantes (siendo mencionado en un $21,9 \%$ de las referencias) mas no así por la comunidad de memoria, la cual lo clasifica como uno más bien secundario. Si bien, este valor se constituye como central desde la perspectiva de los visitantes, es interesante destacar que, tanto en las reseñas online como en la planificación estratégica del parque, este se reconoce como un punto de partida desde donde emergen otros valores centrales tales como el asociativo y el conmemorativo.

Lugar histórico, recuerda la brutalidad de una Dictadura y la memoria de torturados y detenidos desaparecidos. (G-170.)

Es un lugar histórico donde se protagonizó tortura de militares contra ciudadanos y ciudadanas chilenas en tiempos de dictadura militar. (T-33).

luego del Golpe de Estado de 1973, Villa Grimaldi pasa a poder de la Dirección de Inteligencia Nacional (DINA), que la transforma en su Cuartel Terranova, siendo el centro de detención, tortura y exterminio más importante del país. (CMN, 2004).
Otro valor ampliamente reconocido por los visitantes es el valor asociativo, el cual se define por el vínculo que existe entre Villa Grimaldi, su pasado reciente y las violaciones a los derechos humanos cometidas en el sitio. Como ya se mencionó, este valor es constitutivo de los sitios de memoria (ICSC, 2018), no obstante, nuevamente se reconoce como un valor de carácter más bien secundario por parte de la CPPVG.

En este contexto, si se observan los valores centrales reconocidos por la CPPVG, estos corresponden principalmente al valor conmemorativo y al educacional. Si entendemos que la planificación estratégica es redactada por la comunidad de memoria - es decir, sobrevivientes y familiares de las víctimas de Villa Grimaldi- y por los gestores que trabajan en el sitio, se podría especular que estos son los roles que estas comunidades esperan que Villa Grimaldi cumpla tanto para ellos como para el resto de la sociedad, los cuales son expresados en de la misión del sitio el cual busca

recuperar, preservar y poner en valor la memoria histórica de Villa Grimaldi (...) y las memorias vinculadas a las víctimas de las acciones de terrorismo de Estado perpetradas en el lugar (...) y promover una cultura de derechos humanos como base de la convivencia democrática de nuestra sociedad (CPPVG, 2018, p. 7).

Esto debido a que el rol educativo de un sitio de memoria como Villa Grimaldi permite que los visitantes aprendan, a través de la experiencia de la visita, acerca de los hechos ocurridos, tanto en el sitio como a nivel nacional durante la dictadura cívico-militar. De igual modo, educar con respecto a las atrocidades del pasado promueve la reflexión en torno a la importancia de una cultura de los derechos humanos, previniendo la repetición del horror de aquellos años (Mora, 2013).

Por otra parte, el valor conmemorativo es una de las principales razones por las que un sitio como Villa Grimaldi existe, pues funciona como cenotafio (Donetch et al., 2016), el cual permite recordar a aquellos que por ahí pasaron y de los que hasta hoy no se tiene certeza de 
su destino. Los sitios de memoria, entonces, funcionan como un memorial al ausente, "una manera de decirle (...) que es recordado y que no ha muerto en la memoria de sus familiares y amigos" (Piper y Hevia, 2012, p. 19). Este valor es constatado en la presencia de flores y fotografías en el sitio, en la conmemoración de fechas significativas como el 11 de septiembre y también en las siguientes reseñas de los visitantes:

Lugar de mucho dolor, se respira el aire de nuestros compañeros y compañeras que vivieron las atrocidades del fascismo. (F-19).

Hermoso espacio para la memoria y recuerdo de nuestr@s compañer@s caídos en dictadura. (G-26).

Llama la atención que los valores centrales son los únicos reconocidos por el Estado a través del CMN. Esta situación hace referencia al contexto político y social en el que se recuperó y patrimonializó Villa Grimaldi, el cual definió la relación entre el Estado, la comunidad de memoria y la sociedad civil en general. Esta relación originalmente estuvo marcada por el nulo apoyo estatal, evidenciado en la poca participación de ciertos actores políticos con el sitio. Este ha ido mejorando a lo largo del tiempo con la visita de la presidenta Michelle Bachelet a Villa Grimaldi en el año siendo la primera mandataria en hacerlo (Dalla Porta y Gatica, 2006). Desde el gobierno de Bachelet, el financiamiento a diversos sitios de memoria, entre ellos Villa Grimaldi, se concretó. Sin embargo, este involucramiento del Estado en temas de memoria, no se ve reflejado en un cambio de visión de lo que este sitio representa y de los valores que encarna.

\section{Valores disonantes}

Contrario a los valores centrales, donde existe un consenso sobre cuáles son reconocidos transversalmente por las comunidades, hay otros que presentan considerables diferencias entre los distintos actores sociales, siendo algunos altamente percibidos por un grupo y no así por otros. Estas discrepancias en la valorización son lo es lo que se denomina la disonancia del patrimonio, la cual está dada por la diversidad de significados asociados al patrimonio, que normalmente causan desacuerdos sobre cómo el pasado está siendo interpretado y representado por diferentes actores (Kisić, 2016).

Entre estos valores se encuentra, en primer lugar, el valor social. Este es dado por la habilidad de un sitio para promover las conexiones, redes y otras relaciones sociales (Mason, 2002). Es claramente reconocido y expresado por la comunidad de memoria dentro de los objetivos de la planificación estratégica de Villa Grimaldi, la cual propone "desarrollar estrategias para (...) la vinculación con el territorio y con diferentes actores públicos y movimientos sociales y de derechos humanos de interés" (CPPVG, 2018, p. 8). No obstante, este valor no es reconocido por el CMN y las referencias hechas por los visitantes son mínimas (0,08\%).

Otro fenómeno interesante es lo observado con respecto al valor estético. Este, a diferencia del valor social, es bastante reconocido por los visitantes y no así por el resto de las comunidades, las cuales no lo mencionan. Este fenómeno está directamente relacionado con el proyecto original de diseño del parque, el cual, ante la carencia de vestigios materiales, recurrió a la utilización de elementos simbólicos $\mathrm{y}$, posteriormente, a la reconstrucción de ciertas estructuras características del cuartel Terranova. A pesar de que este valor es evidenciado por los visitantes, ha sido duramente criticado por diversos autores a lo largo del tiempo (Lazzara, 2003; Schindel, 2009) y también por la misma comunidad de memoria, la cual se ha referido a la construcción de la memoria del Parque por la Paz de la siguiente manera:

En realidad, las mujeres estábamos... no estábamos de acuerdo con una Villa tan simbólica. Siempre dijimos que era imposible mantener la memoria con una Villa con puras lucecitas, con arbolitos y con pasadizos, que nosotras creíamos que había que reconstruir algunos elementos, por mucho que se creyeran del horror, pero que era importantes porque las generaciones futuras no iban a entender con puros símbolos. (CPPVG, 2011).

En otras palabras, para ciertos grupos -específicamente aquellos directamente relacionados con el sitio, además 
de los académicos dedicados al estudio de los sitios de memoria- el embellecimiento y el simbolismo del parque resultaba inadecuado pues, argumentaban, dificultaba una conexión emocional e intelectual con la memoria del sitio (Taylor, 2011). Si bien, el aspecto estético del Parque por la Paz Villa Grimaldi puede parecer inadecuado para algunos, es ampliamente reconocido y valorado por sus visitantes, pues es percibido como un atenuante del impacto emocional que significa visitar este lugar (Fernández et al., 2018). El contacto con la naturaleza es un estímulo que se contrapone a la memoria de los horrores que ocurrieron en el sitio, el cual puede percibirse como un mensaje de paz y esperanza.

Por último, dentro de los valores disonantes de Villa Grimaldi, se encuentra también la percepción de que el sitio no posee valor y que, por ende, no debería ser considerado parte del patrimonio cultural del país. En este sentido, la categoría de "sin valor" es en sí misma disonante, pues se basa en la concepción de la carencia de valor de algo que es socialmente valorado. Esta valorización de Villa Grimaldi se asocia en general con aquellos grupos negacionistas de las atrocidades cometidas durante la dictadura de Augusto Pinochet y, por lo mismo, presenta bajos niveles de empatía con lo ocurrido en el sitio, los cuales son expresadas bajo consignas como:

Esto es un engaño a los turistas el lugar es sumamente peligroso y de parque por la paz no tiene nada porque es un memorial COMUNISTA!!! (T-27).

Este tipo de juicio no solo expone que estos sitios carecen de significancia e importancia para el país, sino que su mera existencia constituye un peligro para el resto de la sociedad. Asimismo, este tipo de percepción, en facciones más extremas, ha decantado en la vandalización de los sitios de memoria, hecho frecuente en Villa Grimaldi en los últimos años.

\section{Valores secundarios}

Los valores secundarios son todos aquellos que son reconocidos por una sola comunidad, en este caso específicamente por los visitantes, y con un bajo índice de referencia. Desde la perspectiva de la conservación del sitio, estos valores al no ser prioritarios son "sacrificables" por el bien de los valores centrales (Mason, 2002). A continuación, se describirán algunos de ellos.

En primer lugar, se encuentra el valor inspiracional, el cual se define como la capacidad que posee un objeto de generar un impulso de hacer el bien. En el caso de los sitios de memoria como Villa Grimaldi, este puede ser entendido como el objetivo final que tiene el valor educacional, el cual busca promover una respuesta empática en relación con las perpetraciones cometidas en el lugar, fortaleciendo la condena a estos hechos e impulsando la internalización del "Nunca más”, como queda señalado en las siguientes reseñas:

estar ahí y saber que en otro tiempo fue un campo de detención es estremecedor. Igual recomiendo su visita, es algo que la gente tiene que sentir en carne propia para empezar a imaginarse el horror que nunca más debe repetirse. (T-4).

El segundo valor secundario es el espiritual, el cual radica en los significados intangibles que se le atribuyen al sitio y que lo dotan de cierta identidad espiritual. Este puede ser concebido como un carácter sagrado por algunos o como un significado asociado a la esperanza y a la paz para otros. El aspecto espiritual del parque, tal como ocurre con el valor estético, ha sido reconocido por los visitantes como catalizador para la reflexión, ambos pueden ayudar a decantar aquellas fuertes emociones relacionadas con el dolor, tal como se señala en la reseña a continuación:

Lugar sagrado de memoria, justicia y reparación para nuestra sociedad y por la construcción de valores verdaderos, como la Paz, el Amor y la Verdad. Aquí se hace la Alquimia sanadora de la historia. (F-56).

Por otra parte, llama la atención que el valor arqueológico se encuentre dentro de la categoría de valor secundario y, por ende, sea "sacrificable", más aún que sea solo reconocido por los visitantes y no por la comunidad de 
memoria o el Estado, cuando diversos informes nacionales e internacionales lo destacan como fuente de evidencias para procesos judiciales presentes y futuros (Comisión Interamericana de Derechos Humanos, 2019; IPPDH, 2012). En el caso de Villa Grimaldi, si bien los restos arqueológicos son escasos, resultan de vital importancia para la comprensión del lugar y su historia; algunos ejemplos son las gradas de la excasona, el monumento a los rieles y los testimonios de los sobrevivientes, que son considerados de gran importancia por los visitantes. El rol testimonial de Villa Grimaldi, a través de los relatos orales de sus sobrevivientes, promueve la empatía, ya que funciona como evidencia de la verdad, según expresa una de las reseñas:

Realicé una visita guiada por dos personas que estuvieron detenidas cuando Villa Grimaldi fue un centro de tortura, y la experiencia fue impactante. Escuchar en primera persona las atrocidades que allí se cometían, te conecta con una parte lamentable de nuestra historia. (T-54).

Debido a la importancia que tiene el rol testimonial de Villa Grimaldi, es que la CPPVG creó en el año 2005 el Archivo Oral, el cual tiene una doble función: 1) preservar los testimonios para las futuras generaciones, reconociendo este valor como un aspecto intangible del carácter patrimonial de Villa Grimaldi y 2) conectar con las nuevas generaciones a través de los relatos de sus sobrevivientes.

Por último, existen una serie de valores secundarios que están directamente relacionados con la condición de parque que tiene Villa Grimaldi, tales como el recreacional, medioambiental y escénico. Estos dan cuenta de la visión de ciertos visitantes que aprecian el sitio en cuanto área verde dentro de la comuna de Peñalolén y no en su calidad de sitio de memoria. En otras palabras, estos valores reconocidos reflejan un rol secundario que posee Villa Grimaldi, que va más allá de su función memorial y educativa, y que viene a suplir una carencia local: la falta de áreas verdes en el sector, pudiendo ser una oportunidad para conectar con la comunidad local.

\section{Discusión}

Los valores de un sitio de memoria, tal como lo demuestran los resultados de este estudio, no son estáticos ni consensuados, más bien, al ser socialmente construidos, se constituyen de manera subjetiva y varían social y temporalmente. Debido a los altos niveles de contestación en estos sitios, esta variación en los valores es especialmente significativa e incluso, en algunos casos, conflictiva. A partir del análisis de datos, se puede corroborar la subjetividad y multiplicidad de valores atribuidos a un sitio de memoria, como es Villa Grimaldi, por sus comunidades. Bajo la premisa de que un sitio histórico no posee un valor único e inmutable, sino que tiene muchos valores que se traslapan entre ellos (Impey, 2006, citado en Worthing, \& Bond, 2008), el hecho de que las comunidades de Villa Grimaldi reconozcan esta multiplicidad refuerza la idea de la significancia del sitio y que, por lo mismo, merece ser considerado y protegido como parte del patrimonio cultural de Chile. Sin embargo, esta multiplicidad de valores, como se pudo comprobar en el análisis, significa que las distintas comunidades considerarán el sitio valioso por diferentes razones, las cuales pueden ser incluso contradictorias. Asimismo, se reconoce la necesidad de incorporar las diversas miradas en la valorización e interpretación del sitio.

A partir de los resultados, es posible crear un primer esbozo de la significancia cultural de Villa Grimaldi, jerarquizando ciertos valores por sobre otros (Figura 3). Esta significancia cultural reconoce las voces de los diversos actores sociales estudiados y puede dar luces de cómo priorizar la toma de decisiones en la gestión del sitio. La metodología presentada en este estudio, sin embargo, no busca reemplazar metodologías ya adoptadas por los profesionales de Villa Grimaldi, sino que pretende aportar nuevas miradas en torno a la puesta en valor del sitio y, por ende, dar mayor sustento al trabajo ya realizado por la CPPVG.

Si bien este esquema permite jerarquizar los valores reconocidos en él, cumpliendo con lo recomendado por Mason (2002), los resultados del análisis arrojan que, tanto valores como comunidades no son elementos aislados 
Figura 3

Esquema de significación cultural de Villa Grimaldi

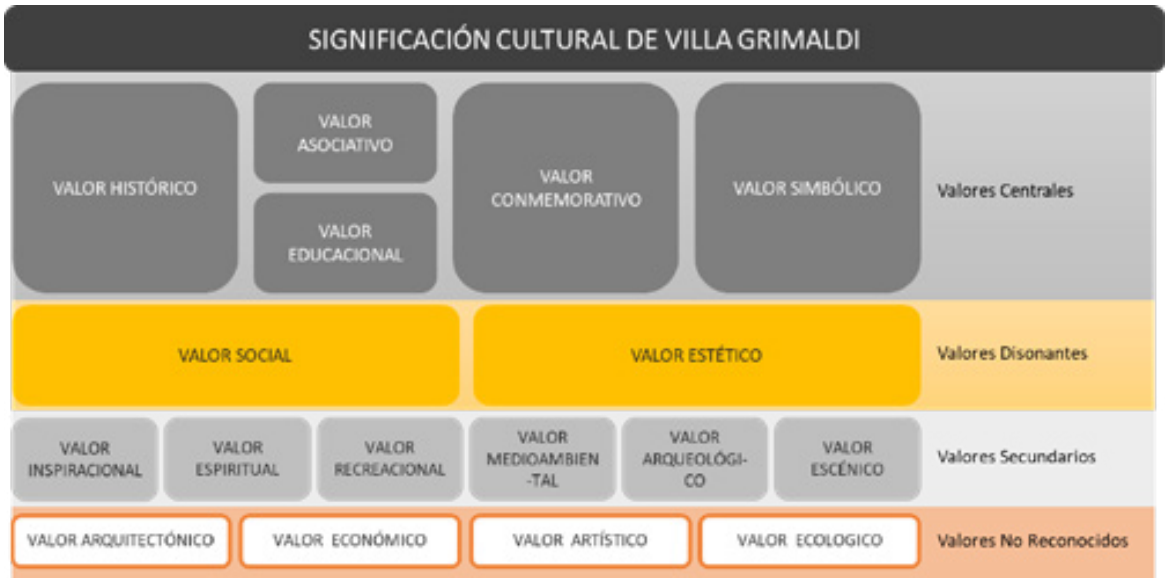

Figura 4

Dinámicas de valores y comunidades en Villa Grimaldi

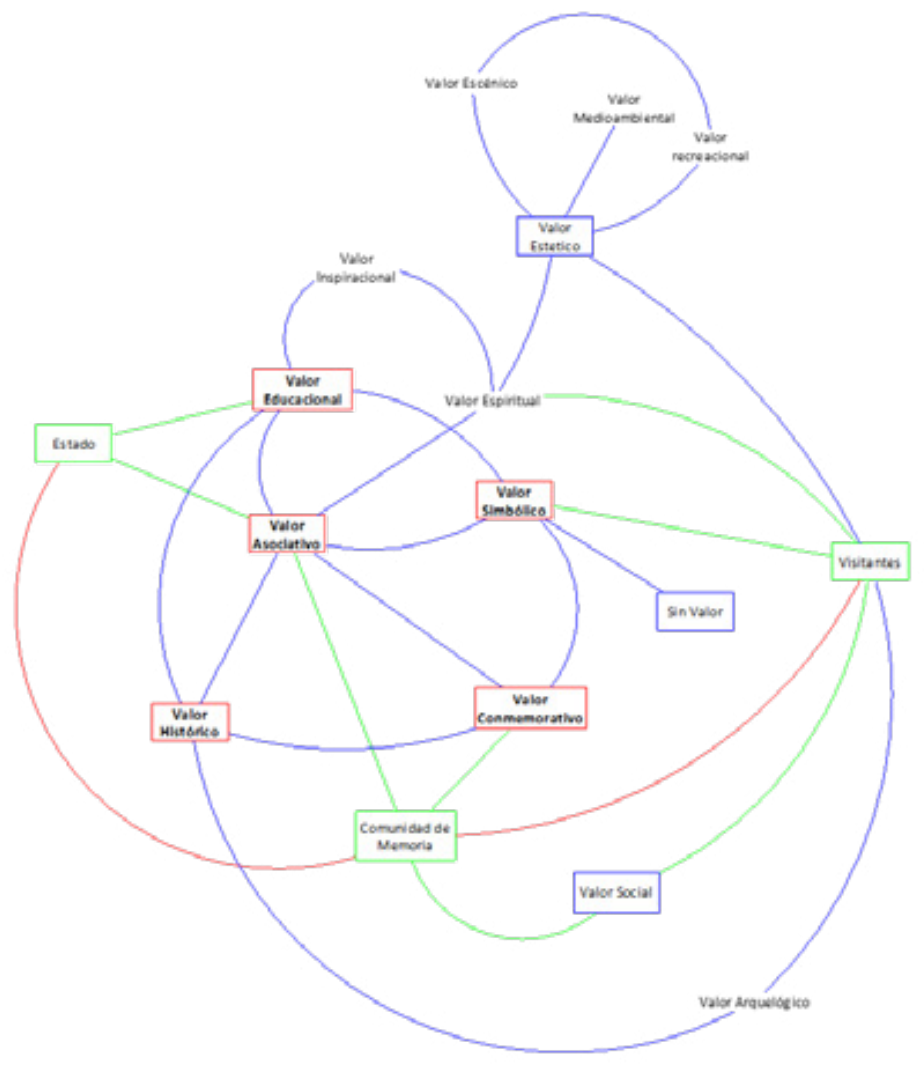

Factores

$\square$ Valor central

Valor disonante

Comunidades
Relaciones

Comunidad-Comunidad

Valor-Valor

Comunidad-Valor 
e independientes, sino todo lo contrario. Las relaciones entre sitio, valores y comunidades son complejas y dependen unas de otras. Por lo mismo, los conceptos de valores "centrales" o "secundarios" parecieran no ser los más aptos. En este sentido, la significancia de un sitio de memoria puede ser entendida como un sistema, el cual es complejo, dinámico y multidimensional (Fouseki, \& Nicolau, 2018), y que, por lo mismo, métodos con un enfoque de sistema son necesarios para comprender los impactos a largo plazo que la toma de decisiones puede tener para las formas en que el sitio es valorado. Por ende, a partir de los resultados del análisis temático se mapearon las relaciones entre comunidades y valores, demostrando la dependencia entre valores y comunidades, las cuales al ser comprendidas pueden mejorar la toma de decisiones (Figura 4).

Villa Grimaldi, en cuanto sitio de memoria, es un espacio contestado y con una fuerte carga política, pues posee un discurso político particular (Hartley, 2012). Por lo mismo, el sitio se encuentra en constantes tensiones ideológicas las cuales no solo dificultan su conservación, sino también la relación que tiene con sus comunidades, lo que produce un efecto contrario al deseado: la percepción de que la narrativa de este lugar es de "unos pocos" y, en un peor escenario, que el lugar no posee valor. Para superar este desafío, los gestores y comunidades de memoria de Villa Grimaldi deben comenzar a incluir dentro del proceso patrimonial, desde la puesta en valor a la toma de decisiones, a las diferentes comunidades periféricas. En este contexto, la significancia cultural de un sitio de memoria debiese ser comprendida como un ejercicio ciudadano, el cual puede fortalecer las relaciones del sitio con las diferentes comunidades, haciéndolas partícipes de su narrativa, educando acerca de la importancia de la memoria, los derechos humanos y la no repetición. Asimismo, se debe entender que la valorización de los sitios de memoria, en cuanto proceso complejo y dinámico, implica que un sitio que es valorado como patrimonio memorial hoy, puede convertirse en un espacio vacío de significado mañana y viceversa (Hevia, 2014). Desde esta perspectiva, su sustentabilidad dependerá principalmente en su capacidad de generar valor entre las diferentes comunidades. En este sentido, una clara comprensión de las diversas comunidades y cómo estas se relacionan con el sitio resulta crucial para desarrollar estrategias de gestión e interpretación que permitan aumentar la conciencia en cuanto al pasado, la implicación de este en el presente y la participación de los actores sociales para construir un nuevo futuro.

Esto resulta particularmente importante a tener en cuenta, sobre todo en el contexto actual del COVID-19 y sus consecuencias, en donde los sectores populares se vieron especialmente afectados. Por su parte en el contexto de los diferentes levantamientos sociales en Chile y el mundo, donde diversos monumentos y bienes patrimoniales han sido cuestionados e interpelados, resulta imperante la inclusión de más comunidades y actores sociales en la puesta en valor del patrimonio, asegurando así su relevancia y sustentabilidad en el futuro.

\section{Conclusiones}

Este artículo proporciona una nueva metodología para estudiar cómo las diferentes comunidades valorizan un sitio de memoria como Villa Grimaldi, la cual puede ser replicada en otros sitios en las distintas etapas de patrimonialización (entendimiento, puesta en valor, gestión, interpretación, etc.). El análisis temático identificó los principales valores que las diferentes comunidades reconocen en el sitio, revelando que estos son altamente contextuales y varían de comunidad en comunidad. En palabras de Lourdes Arizpe (2000):

los valores de un Gobierno van en un camino, los de la elite en otro, diferente a los de la población local, del mundo académico o empresarial. Para reconocer cuál es la mejor estrategia para preservar el patrimonio cultural, necesitamos entender lo que cada uno de estos grupos piensa y las relaciones entre ellos (citado en Seguel, 2017, p. 27).

Por lo mismo, llama la atención la nula referencia a las diferentes comunidades tanto en el decreto del CMN como en el plan de gestión del Parque por la Paz Villa Grimaldi. Especialmente si se tiene en cuenta que la ICSC (2018) recomienda la inclusión de diversos actores 
en la puesta en valor e interpretación del sitio, pues si bien pueden existir diferentes puntos de vista sobre la significancia del lugar, "puede presentar oportunidades para congregar a diversas comunidades con diferentes perspectivas" (ICSC, 2018, p. 17). En este sentido, si bien estos sitios se comenzaron a proteger en una primera instancia por iniciativa de las comunidades de memoria (Seguel, 2019), las cuales exigieron su reconocimiento y protección al Estado como parte de las políticas de memoria y reparación, es necesario incluir a un espectro más amplio de la sociedad, generando una mayor conciencia acerca de la importancia de estos sitios y su memoria.

El caso de estudio muestra que la valorización de un sitio de memoria es un proceso altamente cambiante y extremadamente complejo, el cual está fuertemente integrado en las relaciones entre comunidades y con el sitio. Las diferencias en la valorización están dadas por las percepciones y prejuicios de las diferentes comunidades, las cuales son afectadas por los contextos sociales, políticos, emocionales, etc. Por lo tanto, la puesta en valor de un sitio de memoria como Villa Grimaldi requiere un enfoque integral y holístico.

Primero, es importante la identificación de los distintos grupos sociales que puedan asignar valores al sitio. Se debe comprender que estos pueden ser valorizados por comunidades que no están conectadas directamente con ellos y su historia, las cuales pueden sentirse interpeladas por la narrativa del sitio y las demandas de justicia y reparación. Cada una de ellas puede reconocer múltiples valores, lo cual puede motivarla para validar la importancia de la conservación del patrimonio memorial del país.

En segundo lugar, el diálogo entre estas comunidades puede ayudar a comprender no solo su propia forma de valorizar el sitio, sino que la de otros. Si bien, durante este estudio se analizaron las comunidades y sus valores por separado, se reconoce la interconexión entre ambos factores, los cuales actúan como un sistema complejo y multidimensional, donde cada componente (comunidad y/o valor) soporta y es soportado por los otros. Más aún, el estudio arroja que este sistema se sostiene en el tiempo debido a la relación que existe entre sus elementos. Por lo mismo, sería interesante realizar futuros ejercicios donde las diferentes perspectivas puedan ser congregadas y dialogadas, lo cual podría resultar en una declaración de significancia inclusiva.

Por último, en contextos de fuertes cambios sociales y políticos, donde diversos bienes patrimoniales han sido interpelados, resulta urgente la inclusión de un amplio espectro de la sociedad en los procesos de puesta en valor del patrimonio memorial, acercando a más personas a lo que estos sitios recuerdan, generando mayor conciencia sobre la importancia de una cultura de los derechos humanos. En este sentido, se requieren más investigaciones en torno a los procesos participativos en la puesta en valor, protección e interpretación de los sitios de memoria, los cuales permitan asegurar la sustentabilidad de estos espacios en el futuro.

\section{Referencias bibliográficas}

Australia ICOMOS. (2013). The Burra Charter, The Australia ICOMOS Charter for places of cultural significance. Edición del autor. https://australia.icomos.org/wp-content/uploads/ The-Burra-Charter-2013-Adopted-31.10.2013.pdf

Avrami, E., Macdonald, S., Mason, R., \& Myers, D. (Eds). (2019). Values in heritage management. Emerging approaches and research directions. The Getty Conservation Institute.

Avrami, E., \& Mason, R. (2019). Mapping the issues of values. En E. Avrami, S. Macdonald, R. Mason, \& D. Myers (Eds.), Values in heritage management. Emerging approaches and research directions (pp. 9-33). The Getty Conservation Institute.

Avrami, E., Mason, R., \& De la Torre, M. (2000). Values and Heritage Conservation: Research Report. https://www.getty. edu/conservation/publications_resources/pdf_publications/ values_heritage_research_report.html

Bianchini, M.C. (2014). When memory becomes heritage: Experiences from Santiago, Chile. Culture \& History Digital Journal, 3(2), e023. https://doi.org/10.3989/chdj.2014.023 
Bianchini, M. C. (2015). De la represión al patrimonio: vestigios de la violencia de Estado en Madrid y Santiago de Chile. Revista de Dialectología y Tradiciones Populares, 70(2), 399-426. https://doi.org/10.3989/rdtp.2015.02.005

Braun, V., \& Clarke, V. (2006). Using thematic analysis in psychology. Qualitative Research in Psychology, 3(2), 77-101. https://doi.org/10.1191/1478088706qp063oa

Bustamante, J. (2016). Procesos de activación y patrimonialización de sitios de memoria en Chile. 1990 al presente. Aletheia, 7(13). http://www.memoria.fahce.unlp.edu.ar/ library? $\mathrm{a}=\mathrm{d} \& \mathrm{c}=\mathrm{arti} \& \mathrm{~d}=\mathrm{Jp} 7614$

Cabeza, A. (2017). Introducción al patrimonio de los derechos humanos en Chile. En A. Cabeza, A.P. Cárdenas, P. Seguel y J. Bustamante (Eds.), Patrimonio de la memoria de los derechos humanos en Chile. Sitios de memoria protegidos como Monumentos Nacionales 1996/2016 (pp. 11-18). Consejo de Monumentos Nacionales de Chile.

Clark, K. (2010). Values in cultural resource management. En G., Smith, P. Messenger, \& H. Soderland (Eds.), Heritage values in conterporary society (pp. 89-99). Left Coast Press.

Comisión Interamericana de Derechos Humanos. (2019). Principios sobre Políticas Públicas de Memoria en las Américas. Edición del autor.

Comisión Nacional Sobre Prisión Política y Tortura. (2005). Informe de la Comisión Nacional Sobre Prisión Política y Tortura. Edición del autor.

Corporación Parque por la Paz Villa Grimaldi. (2018). Planificación estratégica 2018-2028. Edición del autor. http://villagrimaldi.cl/wp-content/uploads/2017/11/ Propuesta-Planificacio\%CC\%81n-Estrate\%CC\%81gicaVG-2018-2028-Final.pdf

Corporación Parque por la Paz Villa Grimaldi. (2019). Memoria y balance 2019. Edición del autor. http://villagrimaldi. cl/wp-content/uploads/2021/07/CPPVG-Memoria-yBalance-2019.pdf
Dalla Porta, C. y Gatica, E. (3-5, noviembre, 2016). Las tensiones de la memoria: desafíos y resistencias a veinte años de la recuperación de Villa Grimaldi [Sesión de conferencia]. IX Seminario Internacional Políticas de la Memoria, Buenos Aires, Argentina.http://conti.derhuman.jus.gov.ar/2016/11/ seminario/mesa_30/dallaporta_gatica_mesa_30.pdf

De la Torre, M., MacLean, M., Mason, R., \& Myers, D. (Eds.). (2005). Heritage values in site management. Four case studies. The Getty Conservation Institute.

Decreto N² 264 de 2004 [Ministerio de Educación]. Declara Monumento Histórico el Parque por la Paz Villa Grimaldi. 27 abril 2004. https://www.monumentos.gob.cl/ monumentos/monumentos-historicos/parque-pazvilla-grimaldi

Del Valle, N. (2018). Memorias de la (pos)dictadura: prácticas, fechas y sitios de memoria en el Chile reciente. Revista Mexicana de Ciencias Políticas y Sociales, 63(232), 301-322. https://doi.org/10.22201/ fcpys.2448492xe.2018.232.58111

Díaz-Andreu, M. (2017). Heritage values and the public. Journal of Community Archaeology \& Heritage, 4(1), 2-6. https://doi.org/10.1080/20518196.2016.1228213

Donetch, I., Mosalve, J. y Orellana, C. (2016). No olvidar recordar: puesta en valor de los Sitios de Memoria en Chile [Trabajo final diplomado]. Pontificia Universidad Católica de Chile, Santiago, Chile.

English Heritage (2008). Conservation principles, policies and guidancefor the sustainable management of the historic environment. Edición del autor.

Fernández, R., López, L. y Piper, I. (2018). Recordar la dictadura chilena visitando lugares dememoria. Psicología \& Sociedade, 30(0). https://doi.org/10.1590/1807-0310/2018v30179978

Fouseki, K., \& Nicolau, M. (2018). Urban Heritage Dynamics in 'Heritage-Led Regeneration': Towards a Sustainable Lifestyle Approach. The Historic Environment, 9(3-4), 229248. https://doi.org/10.1080/17567505.2018.1539554 
Fouseki, K., \& Sakka, N. (2013). Valuing an Ancient Palaestra in the Centre of Athens: The Public, The Experts and Aristotle. Conservation and Management of Archaeological Sites, 15(1), 30-44. https://doi.org/10.1179/135050331 3z.00000000045

González, S. (2018). Vecinos recuperando la memoria: la Cárcel de Carabanchel. Scripta Nova, 22, 598. https:// doi.org/10.1344/sn2018.22.21408

Gugglielmucci, A. (2018). Pensar y actuar en red: los lugares de memoria en Colombia. Aletheia, 8(16). http://www. memoria.fahce.unlp.edu.ar/art_revistas/pr.8718/ pr.8718.pdf

Hartley, R. (2012). Signifying the Place of Unforgettable Memory: Atrocity and Trauma in Post-Conflict Landscape. Anthropology Faculty Publications, University of Nebraska-Lincoln. http://digitalcommons.unl.edu/ anthropologyfacpub/58

Hevia, E. (2014). Memorias subterráneas en el Chile actual: el lugar de la traición en las memorias de sobrevivientes de Villa Grimaldi [Tesis de magíster, Universidad de Chile, Santiago, Chile]. Repositorio institucional http:// repositorio.uchile.cl/handle/2250/131417

Instituto de Políticas Públicas en Derechos Humanos del Mercosur. (2012). Principios fundamentales para las políticas públicas sobre sitios de memoria. Edición del autor. https://www.ippdh.mercosur.int/principiosfundamentales-para-las-politicas-publicas-en-materiade-sitios-de-memoria/

International Coalition of Sites of Conscience. (2018). Interpretation of Sites of Memory. Unesco. https:// www.sitesofconscience.org/en/2020/06/the-past-ispresent-sites-of-conscience-in-solidarity/

Kisić, V. (2016). Governing heritage dissonance. Promises and realities of selected cultural policies. European Cultural Foundation.
Korostelina, K. (2019). Understanding values of cultural heritage within the framework of social identity conflicts. En E. Avrami, S. Macdonald, R. Mason, \& D. Myers. (Eds.), Values in heritage management. Emerging approaches and research directions (pp. 83-96). The Getty Conservation Institute.

Lazzara, M. (2003). Tres recorridos de Villa Grimaldi. En E. Jelín y V. Langland (Eds.), Monumentos, memoriales y marcas territoriales (pp. 127-147). Siglo XXI Editores.

Ley N 17.288 de 1970. Sobre Monumentos Nacionales. 04 febrero 1970. Diario Oficial de la República de Chile, número 27.563. https://www.bcn.cl/leychile/ navegar?idNorma $=28892$

Londres 38. (2019). Informe de gestión 2019. Edición del autor. https://www.londres38.cl/1937/articles-101633_ archivo_00.pdf

López, L. (2013). Lugares de memoria de la represión, Contra punto entre dos ex centros de detención recuperados en Chile y Argentina: Villa Grimaldi y el Olimpo [Tesis de magíster, Universidad de Chile, Santiago, Chile]. Repositorio institucional http://repositorio.uchile.cl/ handle/2250/117179

Mason, R. (2002). Assessing values in conservation planning: methodological issues and choices. En M. De la Torre (Ed.), Assessing the values of cultural heritage (pp. 5-30). The Getty Conservation Institute.

McClelland, A., Peel, D., Lerm Hayes, C.M., \& Montgomery, I. (2013). A value-based approach to heritage planning: raising awareness of the dark side of destruction and conservation. The Town Planning Review, 84(5), 583-603. https://doi.org/10.3828/tpr.2013.31

Montenegro, M., Piper, I., Fernández, R. y Sepúlveda, M. (2015). Experiencia y materialidad en lugares de memoria colectiva en Chile. Universitas Psychologica, 14(5), 17231734. https://doi.org/10.11144/javeriana.up14-5.emlm 
Mora, Y. (2013). Lugares de memoria: entre la tensión, la participación y la reflexión. Panorama, 7(13), $97-$ 109. https://webcache.googleusercontent.com/ search?q=cache:064QJUaUNn4J:https://dialnet. unirioja.es/descarga/articulo/4780112.pdf $+\& c d=1$ \&hl=es\&ct=clnk\&gl=uk\&client=firefox-b-d

Newton, D., \& Fouseki, K. (26-27 de septiembre de 2018). Heritage values as a driver or obstacle for energy efficiency in Victorian and Edwardian buildings [Sesión de conferencia]. The 3rd International Conference on Energy Efficiency in Historic Buildings, Visby, Suecia. http:// urn.kb.se/resolve?urn=urn:nbn:se:uu:diva-379646

Nora, P. (2009). Pierre Nora en Les lieux de mémoire. LOM Ediciones

Piper, I. y Hevia, E. (2012). Espacio y recuerdo: archipiélago de memorias en Santiago de Chile. Ocho Libros Editores.

Piper, I., Montenegro, M., Fernández, R., \& Sepúlveda, M. (2018). Memory sites: visiting experiences in Santiago de Chile. Memory Studies, 11(4), 455-468. https://doi. org/10.1177/1750698017693667

Poulios, I. (2014). Discussing strategy in heritage conservation: Living heritage approach as an example of strategic innovation. Journal of Cultural Heritage Management and Sustainable Development, 4(1), 16-34. https://doi. org/10.1108/jchmsd-10-2012-0048

Rebolledo, D. y Sagredo, O. (2020). ¿Cómo presentar a los represores en un sitio de memoria? El caso del Parque por la Paz Villa Grimaldi. Atenea, 521, 211-229. https:// doi.org/10.29393/at521-15cdrh20015

Reyes, M. J., Cruz, M. A., y Aguirre, F. (2016). Los lugares de memoria y las nuevas generaciones: algunos efectos políticos de la transmisión de memorias del pasado reciente de Chile. Revista Española de Ciencia Política, (41), 93-114. https://doi.org/10.21308/recp.41.04

Riegl, A. (1903) El culto moderno a los monumentos. Visor Distribuciones.
Rishi, M., \& Gaur, S.S. (2012). Emerging sales and marketing challenges in the global hospitality industry. A thematic analysis of customer reviews from the world's top two tourist destinations. World Hospitality and Tourism Themes, 4(2), 131-149. https://doi. org/10.1108/17554211211217316

Rosenberg, T. J. (2011). History Museums and Social Cohesion: Building Identity, Bridging Communities, and Addressing Difficult Issues. Peabody Journal of Education, 86(2), 115-128. https://doi.org/10.1080/0161956x.2011.561171

Sagredo, O. (2019). "Fragmentos de memorias rescatadas". Proceso participativo de definición de criterios para la restauración del Muro de Mosaicos de Villa Grimaldi, en Chile. Intervención, 10(19), 77-84. https://doi. org/10.30763/intervencion.2019.19.210

Salazar, G. y Artaza, P. (2013). Villa Grimaldi (Cuartel Terranova): historia, testimonio, reflexión. LOM Ediciones

Schindel, E. (2009). Inscribir el pasado en el presente: memoria y espacio urbano. Política y Cultura, (31). http://www.scielo.org.mx/scielo.php?script=sci_ arttext\&pid=S0188-77422009000100005

Seguel, P. (2017). La dimensión política y social de los procesos de puesta en valor del patrimonio de la memoria de las violaciones a los Derechos Humanos en Chile. 1996-2016. En A. Cabeza, A.P. Cárdenas, P. Seguel y J. Bustamante (Eds.), Patrimonio de la memoria de los derechos humanos en Chile. Sitios de Memoria protegidos como Monumentos Nacionales 1996/2016 (pp. 25-35). Consejo de Monumentos Nacionales de Chile.

Seguel, P. (2018). Las políticas de protección patrimonial de Sitios de Memoria en Chile, 1996-2018. Aproximaciones desde un campo en construcción. Persona y Sociedad, 32(1), 63-97. https://personaysociedad.uahurtado.cl/ index.php/ps/article/view/132

Seguel, P. (2019). Derechos humanos y patrimonio. Historias/ memorias de la represión (para)estatal en Chile. Ediciones del Servicio Nacional del Patrimonio Cultural. 
Sepúlveda, M., Sepúlveda, A., Piper, I. y Troncoso, L. (2015). Lugares de memoria y agenciamientos generacionales: lugar, espacio y experiencia. Última Década, 23(42), 93113. https://doi.org/10.4067/s0718-22362015000100005

Sevcenko, L. (2010). Sites of conscience: new approaches to conflicted memory. Museum International, 62(1-2), 2025. https://doi.org/10.1111/j.1468-0033.2010.01720.x

Smith, L. (2006). Uses of heritage. Routledge.

Smith, L. (2021). Emotional heritage. Visitor engagement at museums and heritage sites. Routledge.

Stern, S. (2004). Remembering Pinochet's Chile: On the eve of London 1998. Duke University Press.

Taylor, D. (2011). Memory, trauma, performance. Aletria Revista de Estudios de Literatura, 21(1), 67-76. https://www. researchgate.net/publication/287730583_Memory_ trauma_performance

Tumbridge, J., \& Ashworth, G. (1996). Dissonant Heritage. The management of the past as a resource in conflict. John Wiley \& Sons Ltd.

Van der Hoeven, A. (2020). Valuing Urban Heritage Through Participatory Heritage Websites: Citizen perceptions of Historic Urban Landscapes. Space and Culture, 23(2), 129-148. https://doi.org/10.1177/1206331218797038

Whigham, K. (2017). Remembering to Prevent: The Preventive Capacity of Public Memory. Genocide Studies and Prevention: An International Journal, 11(2), 53-71. https:// doi.org/10.5038/1911-9933.11.2.1447

Worthing, D., \& Bond, S. (2008). Managing Built Heritage. The Role of Cultural Significance. Blackwell Publishing. 